September 17, 2018

\title{
Entanglement asymmetry for boosted black branes and the bound
}

\author{
Rohit Mishra And Harvendra Singh \\ Theory Division, Saha Institute of Nuclear Physics \\ 1/AF Bidhannagar, Kolkata 700064, India
}

\begin{abstract}
We study the effects of asymmetry in the entanglement thermodynamics of CFT subsystems. It is found that 'boosted' $\mathrm{D} p$-brane backgrounds give rise to the first law of the entanglement thermodynamics where the CFT pressure asymmetry plays a decisive role in the entanglement. Two different strip like subsystems, one parallel to the boost and the other perpendicular, are studied in the perturbative regime $T_{\text {thermal }} \ll T_{E}$. We mainly seek to quantify this entanglement asymmetry as a ratio of the first order entanglement entropies of the excitations. We discuss the AdS-wave backgrounds at zero temperature having maximum asymmetry from where a bound on entanglement asymmetry is obtained. The entanglement asymmetry reduces as we switch on finite temperature in the CFT while it is maximum at zero temperature.
\end{abstract}




\section{Introduction}

The AdS/CFT correspondence [1] has been a quite successful idea in string holography. It relates conformal field theries living on the boundary of anti de Sitter (AdS) spacetime with the supergravity theory in the bulk. The holographic idea of entanglement entropy has also been a focus of recent study in string theory [2]. It has led to some understanding of entanglement entropy in strongly coupled quantum mechanical systems, particularly theories which exhibit scaling property near the critical points [3]. One significant observation has been that the small excitations of the subsystem in the boundary CFT follow entanglement thermodynamic laws somewhat similar to the black hole thermodynamics [4, 5], see also related works [6], [8], [7]. These calculations have become possible now because entanglement entropy can be studied by using gauge/gravity holography [2], that is by evaluating the geometrical area of some spatial extremal sufaces embedded inside asymptotically AdS geometry. It has been proposed recently in [4] that the entanglement entropy $\left(S_{E}\right)$ and the energy of excitations $(\mathcal{E})$ in a pure AdS background give rise to a thermodynamic relation

$$
\triangle \mathcal{E}=T_{E} \triangle S_{E}+\mathcal{V} \triangle \mathcal{P}+\mu_{E} \triangle N
$$

This isn described as the first law of entanglement thermodynamics [4, 5], while the charge and chemical potential contributions can arise in the boosted black-brane cases [9]. These charge excitations could be the Kaluza-Klein (momentum) modes along the compact circle or the winding modes of string wrapped along a T-dual circle.

In this paper we particularly study the effects of boost (excitations) in asymptotically AdS spacetimes on the nature of entanglement first law. We find that the boosted black branes give rise to an asymmetry in the entanglement first law. We study two types of strip subsystems one parallel to the boost and the other perpendicular to the boost direction. There is difference in the 'entanglement pressure' in two cases such that $\triangle \mathcal{P}_{\perp} \leq \triangle \mathcal{P}_{\|}$. We find that primarily the entanglement pressure is responsible for the differences in the entanglement entropies, $\triangle S_{\perp} \geq \triangle S_{\|}$, in the two cases. The entanglement asymmetry may be quantified as a dimensionless ratio

$$
\begin{aligned}
\mathcal{A} & \equiv \frac{\triangle S_{\perp}-\triangle S_{\|}}{\triangle S_{\perp}+\triangle S_{\|}} \\
& =\frac{\beta^{2} \gamma^{2}}{\left(2+\frac{d+3}{d-1} \beta^{2} \gamma^{2}\right)} \leq \frac{d-1}{d+3}
\end{aligned}
$$

We find that the asymmetry depends only on boost and it is bounded from above. The bound is saturated only for the AdS-wave background, which is the case involving infinite boosts. To obtain these results we resort to a perturbative calculation of the entanglement entropy up to first order, where the ratio $\frac{l}{z_{0}}$, of the strip width $(l)$ to the horizon size $\left(z_{0}\right)$, is kept very small. This hierarchy of length scales can also be thought of in terms of the temperatures as $\frac{T_{\text {thermal }}}{T_{E}} \ll \frac{a_{1}}{2 b_{0} \gamma}$.

The paper is organized as follows. In the second section we first reproduce our earlier results for the perpendicular strip case at first order in the perturbative expansion. 
Then we take up the case when the strip subsytem is parallel to the boost direction. The entanglement laws are obtained at the first order. In section-3 we define entanglement asymmetry ratio. It is found that it depends only on the boost velocity, which is a measurable effect. We also obtain entanglement asymmetry in the AdS-wave case also, in which case asymmetry saturates the upper bound. We extend these results to nonconformal D-brane case also in section-4. Finally we conclude in section-5.

\section{Entanglement from boosted black-branes}

The boosted $A d S_{d+1}$ backgrounds we are interested are given by

$$
d s^{2}=\frac{L^{2}}{z^{2}}\left(-\frac{f d t^{2}}{K}+K(d y-\omega)^{2}+d x_{1}^{2}+\cdots+d x_{d-2}^{2}+\frac{d z^{2}}{f}\right)
$$

with functions

$$
f=1-\frac{z^{d}}{z_{0}^{d}}, \quad K=1+\beta^{2} \gamma^{2} \frac{z^{d}}{z_{0}^{d}}
$$

$z=z_{0}$ is the horizon and $0 \leq \beta \leq 1$ is boost parameter, while $\gamma=\frac{1}{\sqrt{1-\beta^{2}}}$. The boost is taken along $y$ direction. The one-form

$$
\omega=\beta^{-1}\left(1-\frac{1}{K}\right) d t
$$

and $L$ is the radius of curvature of AdS spacetime, which is taken very large in string length units. 1

\subsection{A thin (perpendicular) strip}

We first study the entanglement entropy law for a subsystem on the boundary of the $A d S_{d+1}$ backgrounds (1) where strip is perpendicular to the boost direction: the strip width is $-l / 2 \leq x^{1} \leq l / 2$, while the boost is along $y$ direction. Thus the steps in this section are same as in our presious work [9]. We embed the $(d-1)$-dimensional strip-like (constant $t$ surface) inside the bulk geometry. The two boundaries of the extremal surface coincide with the two ends of the interval $\triangle x^{1}$. The size of the rest of the coordinates, $0 \leq y \leq l_{y}, 0 \leq x^{i} \leq l_{i}$, is taken very large, such that $l_{y}, l_{i} \gg l$. As per the RyuTakayanagi prescription [2] the entanglement entropy of the strip subsystem is given in terms of the geometrical area of the extremal surface (constant time)

$$
S_{\perp} \equiv \frac{[A]_{\text {Strip }}}{4 G_{d+1}}=\frac{V_{d-2} L^{d-1}}{2 G_{d+1}} \int_{\epsilon}^{z_{*}} \frac{d z}{z^{d-1}} \sqrt{K} \sqrt{\frac{1}{f}+\left(\partial_{z} x^{1}\right)^{2}}
$$

where $G_{d+1}$ is $(d+1)$-dimensional Newton's constant (of bulk gravity) and $V_{d-2} \equiv$ $l_{y} l_{2} l_{3} \cdots l_{d-2}$ is the net spatial volume of the strip on the boundary. We will be mainly

\footnotetext{
${ }^{1}$ For example, in the $A d S_{5} \times S^{5}$ near-horizon geometry of $n$ coincident D3-branes, we shall have $L^{4} \equiv 2 \pi g_{Y M}^{2} n$ and the 't Hooft coupling constant $g_{Y M}^{2} n \gg 1$.
} 
working for $d>2$ here. In our notation $z=\epsilon \sim 0$ is the cut-off scale and $z=z_{*}$ is the turning point of extremal surface. In the above area functional $K(z)$, and $f(z)$ are known functions, so we only need to extremize for $x^{1}(z)$. After extremization the entanglement entropy for perpendicular strip subsytem can be written as

$$
S_{\perp}=\frac{V_{d-2} L^{d-1}}{2 G_{d+1}} \int_{\epsilon}^{z_{*}} \frac{d z}{z^{d-1}} \frac{K}{\sqrt{f} \sqrt{K-K_{*}\left(\frac{z}{z_{*}}\right)^{2 d-2}}}
$$

where $\left.K_{*} \equiv K(z)\right|_{z=z_{*}}$. The boundary value $x^{1}(0)=l / 2$ has the integral relation

$$
\frac{l}{2}=\int_{0}^{z_{*}} d z\left(\frac{z}{z_{*}}\right)^{d-1} \frac{1}{\sqrt{f} \sqrt{\frac{K}{K_{*}}-\left(\frac{z}{z_{*}}\right)^{2 d-2}}}
$$

which relates $l$ with the turning point $z_{*}$. The turning-point takes the mid-point value $x^{1}\left(z_{*}\right)=0$ on the boundary.

When strip subsystem is a small the turning point will lie in the proximity of asymptotic boundary region only $\left(z_{*} \ll z_{0}\right)$. We can evaluate the entanglement entropy (5) by expanding it around the AdS (i.e. treating pure AdS as a ground state). We take boost to be finite such that

$$
\frac{z_{*}^{d}}{z_{0}^{d}} \ll 1, \quad \frac{(\beta \gamma)^{2} z_{*}^{d}}{z_{0}^{d}} \ll 1
$$

is always maintained. In this limit we can estimate the entropy perturbatively. Under these approximations, entanglement entropy contribution (above pure AdS) at first order is given by [9] 2

$$
\triangle S_{\perp}=S_{\perp}-S_{A d S}=\frac{L^{d-1} V_{d-2}}{16 G_{d+1}} \frac{a_{1} l^{2}}{b_{0}^{2}}\left(\frac{d-1}{d+1}+\beta^{2} \gamma^{2}\right) \frac{1}{z_{0}^{d}}
$$

The CFT energy and pressure can be obtained by expanding the bulk geometry (1) in Fefferman-Graham coordinates valid near the boundary [15]. The energy of the excitations is

$$
\triangle \mathcal{E}=\frac{L^{d-1} V_{d-2} l}{16 \pi G_{d+1}}\left(\frac{d-1}{d}+\beta^{2} \gamma^{2}\right) \frac{d}{z_{0}^{d}}
$$

The volume is $V_{d-2} \equiv l_{y} l_{2} \cdots l_{d-2}$. The pressure along $y$ direction is

$$
\triangle \mathcal{P}_{\|}=\triangle \mathcal{P}_{y}=\frac{L^{d-1} d}{16 \pi G_{d+1}}\left(\frac{1}{d}+\beta^{2} \gamma^{2}\right) \frac{1}{z_{0}^{d}}
$$

while the pressure along all other $x_{i}$ 's (perpendicular to the boost direction) is identical and is given by

$$
\triangle \mathcal{P}_{\perp}=\frac{L^{d-1}}{16 \pi G_{d+1}} \frac{1}{z_{0}^{d}}=\triangle \mathcal{P}_{1}=\triangle \mathcal{P}_{2}=\cdots
$$

2 The coefficients (given in [9] also) are defined as; $b_{0}=\int_{0}^{1} d \xi \xi^{d-1} \frac{1}{\sqrt{1-\xi^{2 d-2}}}=\frac{1}{2(d-1)} B\left(\frac{d}{2 d-2}, \frac{1}{2}\right) \equiv$ $(2-d) a_{0}, \quad b_{1}=\int_{0}^{1} d \xi \xi^{2 d-1} \frac{1}{\sqrt{1-\xi^{2 d-2}}}=\frac{1}{2(d-1)} B\left(\frac{d}{d-1}, \frac{1}{2}\right) \equiv \frac{2}{d+1} a_{1}$, where $B(m, n)=\frac{\Gamma(m) \Gamma(n)}{\Gamma(m+n)}$ are the Beta-functions. 
This pressure asymmetry is solely due to the boost. For example the pressure is more along the $y$ (boost) direction as compared to $x^{i}$ 's coordinates. Using (9) and (11) we can express eq.(8) as

$$
\triangle S_{\perp}=\frac{1}{T_{E}}\left(\triangle \mathcal{E}-\frac{d-1}{d+1} \mathcal{V}_{\perp} \triangle \mathcal{P}_{\perp}\right)
$$

where $\mathcal{V}_{\perp} \equiv l\left[l_{y} l_{2} \cdots l_{d-2}\right]$ is the net volume of the strip subsystem. The entanglement temperature is given by

$$
T_{E}^{\perp}=\frac{\left(B\left(\frac{d}{2 d-2}, \frac{1}{2}\right)\right)^{2}}{2(d-1) B\left(\frac{1}{d-1}, \frac{1}{2}\right)} \frac{d}{\pi l} .
$$

The temperature is inversely proportional to the width of strip. The equation (12) simply describes the first law of entanglement thermodynamics [5, 4]. Subtle changes will occur in this expression when strip is taken along the boost.

\subsection{Strip along the boost}

We now study the entanglement entropy of a strip subsystem such that its width is parallel to the boost (flow) direction. That is, we take the boundaries of the extremal surface to coincide with the two ends of $\triangle y$ interval: $-l / 2 \leq y \leq l / 2$. The regulated size of rest of the coordinates will be taken much larger $0 \leq x^{i} \leq l_{i}$, such that $l_{i} \gg l(i=1,2, \cdots, d-2) 3$ Taking the constant time slice the entanglement entropy of the parallel strip becomes

$$
S_{\|}=\frac{V_{d-2} L^{d-1}}{2 G_{d+1}} \int_{\epsilon}^{z_{*}} \frac{d z}{z^{d-1}} \sqrt{\frac{1}{f}+K\left(\partial_{z} y\right)^{2}}
$$

where now $V_{d-2} \equiv l_{1} l_{2} \cdots l_{d-2}$ is the spatial volume. The identification of the extremal strip boundary, $y(0)=l / 2$, leads to the integral relation

$$
\frac{l}{2}=\int_{0}^{z_{*}} d z\left(\frac{z}{z_{*}}\right)^{d-1} \frac{1}{\sqrt{f K} \sqrt{\frac{K}{K_{*}}-\left(\frac{z}{z_{*}}\right)^{2 d-2}}}
$$

which relates $l$ with the turning point $z_{*}$ of the strip. The turning-point takes the midvalue $y\left(z_{*}\right)=0$. The final expression of the entanglement entropy for the parallel strip subsystem now becomes

$$
S_{\|}=\frac{V_{d-2} L^{d-1}}{2 G_{d+1}} \int_{\epsilon}^{z_{*}} \frac{d z}{z^{d-1}} \frac{\sqrt{K}}{\sqrt{f} \sqrt{K-K_{*}\left(\frac{z}{z_{*}}\right)^{2 d-2}}}
$$

\footnotetext{
${ }^{3}$ We wish to embed the $\triangle y$ interval, but since the boost is also along $y$, both 'time' $t(z)$ and $y(z)$ would have to be embedded in the bulk in a covariant manner [12. So one has to be a bit cautious while working with stationary metric cases [10] [11. However, it can be explicitly shown that, in the perturbative expansion (for small strips) to know the entropy only upto first order (next to the pure AdS), just taking a constant $t$ slice would suffice. The deviations in extremal surface geometry away from the constant time slice will contribute only to the second order terms in the expansion. Our aim in this work is to know only the first order terms in the expansions of $z_{*}$ and strip area.
} 
Since the parallel system has not been covered in [9] let us provide some essential details perturbative calculation here. In small strip cases, the equation (15) can be expanded perturbatively as

$$
l=2 z_{*}\left(b_{0}+\frac{z_{*}^{d}}{2 z_{0}^{d}}\left(\left(1+\frac{2 \beta^{2} \gamma^{2}}{d-1}\right) b_{1}-\frac{\beta^{2} \gamma^{2}}{d-1} b_{0}\right)\right)+\cdots
$$

where dots indicate terms of higher powers in $\left(\frac{z_{*}}{z_{0}}\right)^{d}$, and various coefficients are defined earlier. From here keeping only up to first order the equation implies

$$
z_{*}=\frac{\bar{z}_{*}}{1+\frac{\bar{z}_{*}^{d}}{2 z_{0}^{d}}\left(\left(1+\frac{2 \beta^{2} \gamma^{2}}{d-1}\right) \frac{b_{1}}{b_{0}}-\frac{\beta^{2} \gamma^{2}}{d-1}\right)}
$$

where $\bar{z}_{*} \equiv \frac{l}{2 b_{0}}$ being the turning point of pure AdS having the same strip width as $l$. Having obtained the turning point expansion, a similar expansion around pure AdS can be made for the area functional also. Suppressing the details, after regularizing the area integral (16), the net change in the area of parallel strip (above pure AdS value) comes out to be

$$
\triangle A_{\|}=\frac{a_{0} \bar{z}_{*}^{2}}{z_{0}^{d}}\left(\frac{a_{1}}{a_{0}}-\left(1-\beta^{2} \gamma^{2}\right) \frac{b_{1}}{a_{0}}\right)
$$

and corresponding change in the entropy for parallel strip becomes

$$
\triangle S_{\|}=\frac{L^{d-1} V_{d-2}}{16 G_{d+1}} \frac{a_{1} l^{2}}{b_{0}^{2}}\left(\frac{d-1}{d+1}+\frac{2}{d+1} \beta^{2} \gamma^{2}\right) \frac{1}{z_{0}^{d}} .
$$

The equation (20) is complete expression up to the first order. The entanglement first law for a strip along the flow becomes

$$
\triangle S_{\|}=\frac{1}{T_{E}^{\|}}\left(\triangle E_{\|}-\frac{d-1}{d+1} \mathcal{V}_{\|} \triangle P_{\|}\right)
$$

where $\mathcal{V}_{\|}=l V_{d-2}=l\left[l_{1} l_{2} \cdots l_{d-2}\right]$, and $\triangle \mathcal{P}_{\|}=\triangle \mathcal{P}_{y}$ is defined earlier. The temperature is

$$
T_{E}^{\|}=\frac{b_{0}^{2}}{a_{1}} \frac{d}{\pi l}=T_{E}^{\perp}
$$

The two temperatures remain the same but the entanglement entropies differ significantly.

\section{Entanglement asymmetry and the bound}

Following from previous section, with out any loss of generality we can always take the volume of the strip subsystems to be equal

$$
\mathcal{V}_{\|}=\mathcal{V}_{\perp}=l . V_{d-2}
$$

This only means that regulated size of the boxes is kept the same in both the cases, along with the strip width $l$. It implies that

$$
T_{E}^{\|}=T_{E}^{\perp}, \quad \triangle \mathcal{E}_{\|}=\triangle \mathcal{E}_{\perp} .
$$


Comparing the two types of entropy results, the difference is given by

$$
\begin{gathered}
\triangle S_{\perp}-\triangle S_{\|}=\frac{L^{d-1} V_{d-2}}{16 G_{d+1}} \frac{a_{1} l^{2}}{b_{0}^{2}}\left(\frac{d-1}{d+1} \beta^{2} \gamma^{2}\right) \frac{1}{z_{0}^{d}} \\
=\frac{d-1}{d+1} \mathcal{V}\left(\triangle \mathcal{P}_{\|}-\triangle \mathcal{P}_{\perp}\right) .
\end{gathered}
$$

The right hand side is a positive definite expression. Hence we can deduce that entanglement entropy is more for a perpendicular strip subsytem as compared to the parallel set-up, eventhough the energy of excitations and entanglement temperatures remain the same for both. The key to this entropy enhancement effect,

$$
\triangle S_{\perp} \geq \triangle S_{\|}
$$

can directly be alluded to unequal entanglement pressure;

$$
\triangle \mathcal{P}_{\perp} \leq \triangle \mathcal{P}_{\|}
$$

Thus more energy is consumed by the excitations in the parallel strip (with an increased pressure) as compared to the perpendicular strip (having a low pressure along the strip). This suggests that in the boundary CFT 'pressure' plays a vital role in determining the entanglement entropy of the subsytems. The equation (25) also implies that, up to first order, the net difference of the entanglement entropies is

$$
S_{\perp}-S_{\|} \quad=\frac{L^{d-1} V_{d-2}}{16 G_{d+1}} \frac{a_{1} l^{2}}{b_{0}^{2}}\left(\frac{d-1}{d+1} \beta^{2} \gamma^{2}\right) \frac{1}{z_{0}^{d}}
$$

Thus the entropy asymmetry coexists with pressure asymmetry in the CFT, whereas $\left.\triangle S_{\perp}\right|_{\beta=0}=\left.\triangle S_{\|}\right|_{\beta=0}$.

We can now define the entanglement asymmetry as a ratio

$$
\mathcal{A} \equiv \frac{\triangle S_{\perp}-\triangle S_{\|}}{\triangle S_{\perp}+\triangle S_{\|}}=\frac{\beta^{2} \gamma^{2}}{\left(2+\frac{d+3}{d-1} \beta^{2} \gamma^{2}\right)}
$$

Thus nonzero boost $(\beta \leq 1)$ will always induce entanglement asymmetry in the boundary CFT. The asymmetry will however vanishes for $\beta=0$. Note that these results have been derived in the perturbative regime described in (7) only up to first order. We also learn that the asymmetry will always be bounded. In the above the bound is saturated only in the large boost limit, which we shall discuss in the next section.

We could however define an entanglement entropy ratio as

$$
\mathcal{R} \equiv \frac{\triangle S_{\|}}{\triangle S_{\perp}}=\frac{1+\frac{2}{d-1} \beta^{2} \gamma^{2}}{1+\frac{d+1}{d-1} \beta^{2} \gamma^{2}} \geq \frac{2}{d+1}
$$

a quantity which depends on the boost only and is devoid of external factors like shape and size. Then

$$
\mathcal{A} \equiv \frac{1-\mathcal{R}}{1+\mathcal{R}} \leq \frac{d-1}{d+3}
$$

We shall show that the bound is saturated in the case of AdS-wave in the next section. The maximum value $\mathcal{R}$ can take is one for which entanglement asymmetry vanishes. 


\section{$3.1 \quad \beta \rightarrow 1, z_{0} \rightarrow \infty$ limit (pressureless system)}

In present examples the pressure in the $\mathrm{CFT}_{d}$ can be controlled by regulating the boost. We now show that there exists a simultaneous double limit in which the pressure asymmetry of the CFT excitations becomes optimal. We take a double limit $\beta \rightarrow 1, z_{0} \rightarrow \infty$, keeping the ratio

$$
\frac{\beta^{2} \gamma^{2}}{z_{0}^{d}}=\frac{1}{z_{I}^{d}}=\text { Fixed }
$$

These double limits has previously been explored in [13] in connection with Lifshitz type backgrounds from black $\mathrm{D} p$ branes (lightcone coordinates). Under these limits the bulk geometry (11) reduces to the following AdS-wave background

$$
d s^{2}=\frac{L^{2}}{z^{2}}\left(-K^{-1} d t^{2}+K\left(d y-\left(1-K^{-1}\right) d t\right)^{2}+d x_{1}^{2}+\cdots+d x_{d-2}^{2}+d z^{2}\right)
$$

with the new function $K=1+\frac{z^{d}}{z_{I}^{d}}$, where $z=z_{I}$ is an scale which determines momentum of the wave travelling in the $y$ direction. (The entanglement of strip systems for AdSwaves has previously been explored by [14] also.) For this background the energy of the excitations in the CFT becomes

$$
\triangle \mathcal{E}=\frac{L^{d-1} V_{d-2} l}{16 \pi G_{d+1}} \frac{d}{z_{I}^{d}}
$$

The pressure along the wave $(y)$ direction is

$$
\triangle \mathcal{P}_{\|}=\triangle \mathcal{P}_{y}=\frac{L^{d-1} d}{16 \pi G_{d+1}} \frac{1}{z_{I}^{d}}
$$

while the pressure along all $x_{i}$ 's (perpendicular to the wave direction) identically vanishes

$$
\triangle \mathcal{P}_{\perp}=0
$$

in the boundary $\mathrm{CFT}_{d}$, which is a conformal theory with traceless energy-momentum tensor.

The double limits can be directly employed on the entropy results obtained in the previous section, provided we maintain $\frac{z_{*}^{d}}{z_{I}^{d}} \ll 1$. Employing the limits on the entropy expressions in eqs. (8) and (20), it gives us

$$
\triangle S_{\perp}=\frac{L^{d-1} V_{d-2}}{16 G_{d+1}} \frac{a_{1} l^{2}}{b_{0}^{2}} \frac{1}{z_{I}^{d}}=\frac{1}{T_{E}}(\triangle \mathcal{E})
$$

while

$$
\triangle S_{\|}=\frac{L^{d-1} V_{d-2}}{16 G_{d+1}} \frac{a_{1} l^{2}}{b_{0}^{2}}\left(\frac{2}{d+1}\right) \frac{1}{z_{I}^{d}}=\frac{1}{T_{E}}\left(\triangle \mathcal{E}-\frac{d-1}{d+1} \mathcal{V} \triangle \mathcal{P}_{\|}\right)
$$

The width $l$ is kept the same in both cases as well as the volumes. Hence entanglement temperatures, $T_{E}=\frac{b_{0}^{2}}{a_{1}} \frac{d}{\pi l}$, and $\triangle \mathcal{E}$, remain the same for both the cases. Particularly 
in the former case there is no entanglement pressure along the strip ( $x^{1}$ direction). As no 'entanglement work' seems to have been done by the excitations due to vanishing pressure, the entropy remains maximal in the perpendicular direction. 4 While in the latter case there is finite pressure along the strip width, so finite energy is consumed by the excitations to work against the pressure as they take part in the entanglement. Thus the work done against entanglement pressure costs finite energy which essentially leads to a reduction in the net entanglement entropy in direction parallel to propagation of the wave.

From equations (37) and (38) for the AdS-wave case the ratio becomes

$$
\mathcal{R}_{\text {wave }}=\frac{\triangle S_{\|}}{\triangle S_{\perp}}=\frac{2}{d+1} .
$$

This is a remarkable relation and is identical to one in (30). It remains true at the linear order in perturbation (over and above the AdS background). At the higher orders in $\frac{z_{*}^{d}}{z_{I}^{d}}$ expansion this result might change, we hope to report on this in our future work [17]. The entanglement asymmetry becomes

$$
\mathcal{A}_{\text {wave }} \equiv \frac{\triangle S_{\perp}-\triangle S_{\|}}{\triangle S_{\perp}+\triangle S_{\|}}=\frac{d-1}{d+3} .
$$

The asymmetry has optimal value and is universal in nature. The relations (39) and (40) are applicable only when $d>2$, because for $d=2$ (i.e. $A d S_{3}$-wave) the analogue of $\triangle S_{\perp}$ does not exist, but the form of entanglement first law as in (38) for parallel strip does hold good.

\section{Non-conformal boosted black D-branes}

The conformal cases of $A d S$ seometries which are near horizon geometries of D3 and M2/M5 branes are covered in the previous section. In this section we wish to extend entanglement asymmetry analysis to the nonconformal $\mathrm{D} p$ brane backgrounds [18]. We are interested in the boosted $\mathrm{D} p$-brane geometry so that suitable asymmetry is generated. These nonconformal backgrounds can be written as

$$
\begin{aligned}
& d s^{2}=g_{e f f}\left[-\frac{f}{z^{2} K} d t^{2}+\frac{K}{z^{2}}(d y-\omega)^{2}+\frac{d x_{2}^{2}+\cdots+d x_{p}^{2}}{z^{2}}+\frac{4}{(5-p)^{2}} \frac{d z^{2}}{z^{2} f}+d \Omega_{8-p}^{2}\right] \\
& e^{\phi}=\frac{(2 \pi)^{2-p}}{d_{p} N} g_{e f f}^{\frac{7-p}{2}}
\end{aligned}
$$

\footnotetext{
${ }^{4}$ The 'entanglement work' or aptly the 'work function' could be defined as: $\Delta \mathcal{W}=l V_{d-2} \triangle \mathcal{P}=l \cdot \mathcal{F}$. The physical origin of entanglement work may be traced to the fact that finite pressure causes some energy for the excitations to work against it. It is always proportional to the size of the strip. In other words, there is a force $\mathcal{F}$ on the strip walls, and it will take $\triangle \mathcal{W}$ amount of energy in moving the walls upto a separation $l$. This in turn implies there will be a suppression of the excitations that can take part in the entanglement. Note these entanglement quantities are purely of non-thermal in nature, as thermal temperature is vanishing for the AdS-wave, correspondingly thermal entropy and thermal pressure are also vanishing.
} 
along with appropriate $F_{(p+2)}$ form Ramond-Ramond flux. The strength of the string coupling depends on effective YM coupling $g_{\text {eff }}=\left(l_{p} z^{3-p}\right)^{\frac{1}{5-p}}$ and the functions are defined as

$$
\begin{aligned}
& f=1-\frac{z^{\tilde{p}}}{z_{0}^{\tilde{p}}}, \quad K=1+\beta^{2} \gamma^{2} \frac{z^{\tilde{p}}}{z_{0}^{\tilde{p}}} \\
& \omega=\beta^{-1}\left(1-\frac{1}{K}\right) d t
\end{aligned}
$$

with $z=z_{0}$ being the location of horizon and $0 \leq \beta \leq 1$ is the boost. The boost is taken along the $y$ direction and geometry along brane directions has asymmetry. The parameters are defined as

$$
l_{p} \equiv d_{p} g_{Y M}^{2} N, \quad \tilde{p}=\frac{14-2 p}{5-p}
$$

where $d_{p}$ is a fixed normalisation factor for a given $p$ brane (The exact expression will not be needed here but it can be found out in [18]). The parameter $l_{p}$ is essentially the 't Hooft coupling constant and it controls the curvature of spacetime which is to be taken small in string length units $\left(l_{s}=1\right)$ and for which $N$ is taken to be large enough. The boosted geometry (41) is conformally $A d S_{p+2} \times S^{8-p}$, a near-horizon geometry of $N$ coincident $\mathrm{D} p$-branes. Only for $p=3$ case the geometry becomes conformal and is discussed earlier. We are discussing the asymmetry cases for that we need $p=2$ or $p=4$, for them at least two asymmetric brane directions are available.

\subsection{Entropy of thin strips}

We first consider a thin strip in a perpedicular direction to the boost, say $x_{2}$. The RyuTakayanagi entropy functional is given by

$$
\begin{aligned}
& S_{\perp}=\frac{V_{p-1} \Theta_{8-p} Q_{p}}{2 G_{N}} \int_{\epsilon}^{z_{*}} \frac{d z}{z^{\tilde{p}-1}} \sqrt{K} \sqrt{\frac{4}{(5-p)^{2}} \frac{1}{f}+\left(\partial_{z} x_{2}\right)^{2}} \\
& =\frac{V_{p-1} \Theta_{8-p}}{2 G_{N}} \frac{2 Q_{p}}{5-p} \int_{\epsilon}^{z_{*}} \frac{d z}{z^{\tilde{p}-1}} \sqrt{K} \sqrt{\frac{1}{f}+\left(\partial_{z} \bar{x}_{2}\right)^{2}}
\end{aligned}
$$

where $Q_{p} \equiv \frac{(2 \pi)^{2 p-4} \sqrt{l_{p}^{\tilde{p}}}}{g_{Y M}^{4}}$ while $\Theta_{8-p}$ is the volume of unit radius $S^{8-p}$ and $G_{N}$ is the 10dimensional Newton's. We shall consider a small legth interval $-\frac{l}{2} \leq \bar{x}_{2} \leq \frac{l}{2}$, but due to the scaling $x_{2}=\frac{2}{5-p} \bar{x}_{2}$ in eq.(44) the actual width of the strip is $\frac{2 l}{5-p}$. One can see that the integrand in the second line in (44) is strikingly same as that for the conformal case discussed earlier, except that parameter $\tilde{p}$ can take fractional values. (For example, for D2-branes $\tilde{p}=\frac{10}{3}$, but for D4-branes $\tilde{p}=6$.) So the rest of the calculations is straight forward: Extremizing the area and making a perturbative expansion keeping the ratio $\frac{l}{z_{0}}<1$, as in previous sections. Avoiding the unnecessary details we quote the result from eq.(8). The entanglement entropy of the excitations above the extremality is

$$
\triangle S_{\perp}=\frac{V_{p-1} \Theta_{8-p}}{16 G_{N}} \frac{2 Q_{p}}{5-p} \frac{a_{1} l^{2}}{b_{0}^{2}}\left(\frac{\tilde{p}-1}{\tilde{p}+1}+\beta^{2} \gamma^{2}\right) \frac{1}{z_{0}^{d}}
$$


where new beta functions are given as $\left.b_{0} \equiv \frac{1}{2(\tilde{p}-1)} B\left(\frac{\tilde{p}}{2 \tilde{p}-2}, \frac{1}{2}\right)\right)$ and $a_{1} \equiv \frac{1}{2(\tilde{p}-1)} B\left(\frac{1}{\tilde{p}-1}, \frac{1}{2}\right)$. We come to conclusion that the entropy of excitations in a nonconformal $(p+1)$-dimensional theory at the first order can be written as

$$
\triangle S_{\perp}=\frac{1}{T_{E}^{\perp}}\left(\triangle \mathcal{E}-\frac{\tilde{p}-1}{\tilde{p}+1} \mathcal{V}_{\perp} \triangle \mathcal{P}_{\perp}\right)
$$

where $\mathcal{V}_{\perp}=\frac{2}{5-p} l V_{p-1}$ is the net volume of the strip subsystem, while the energy and pressure expressions are in appendix. The entanglement temperature is defined by

$$
T_{E}^{\perp}=\frac{\left(B\left(\frac{\tilde{p}}{2 \tilde{p}-2}, \frac{1}{2}\right)\right)^{2}}{2(\tilde{p}-1) B\left(\frac{1}{\tilde{p}-1}, \frac{1}{2}\right)} \frac{(7-p)}{\pi l} .
$$

The temperature is inversely proportional to the width of strip. But compared to the law in (12) subtle changes have occured in the pressure term in (46). Namely the coefficient $\frac{\tilde{p}-1}{\tilde{p}+1}$ in (46) is different from the ratio $\frac{d-1}{d+1}$ which appears in (12). (Note $d$ takes only integer values and is directly correlated with the dimensionality of $A d S_{d+1}$. This cannot be said about $\tilde{p}$.) Let us comment here that for unboosted nonconformal D-brane case the result (46) was first obtained in [6]. So it is interesting to observe that the form of first law with boost excitations remains the same as in unboosted case [6], although all physical quantities have themselves changed.

In the next we consider an strip interval in the direction parallel to the boost, i.e. along $y$ direction. The entropy functional is given by

$$
S_{\|}=\frac{V_{p-1} \Theta_{8-p}}{2 G_{N} g_{Y M}^{4}} \frac{2 Q_{p}}{5-p} \int_{\epsilon}^{z_{*}} \frac{d z}{z^{\tilde{p}-1}} \sqrt{\frac{1}{f}+K\left(\partial_{z} \bar{y}\right)^{2}}
$$

where now $V_{p-1}$ is regulated volume of all the $x^{i}$ coordinates. We have scaled $y=\frac{2}{5-p} \bar{y}$ and taken the width to be $-l / 2 \leq \bar{y} \leq l / 2$. As usual extremizing the strip area and expanding up to first order in the ratio $l / z_{0} \ll 1$, we come to conclusion that the entropy of excitations above extremality for a parallel strip follows the law

$$
\triangle S_{\|}=\frac{1}{T_{E}^{\|}}\left(\triangle \mathcal{E}-\frac{\tilde{p}-1}{\tilde{p}+1} \mathcal{V}_{\|} \triangle \mathcal{P}_{\|}\right)
$$

where $\mathcal{V}_{\|}=\frac{2 l}{5-p} V_{p-1}$ is the net volume of the parallel strip subsystem. Since we have kept the same width $\frac{2 l}{5-p}$ in both the situations, the entanglement temperature are identical

$$
T_{E}^{\|}=T_{E}^{\perp}
$$

Now if we set $\mathcal{V}_{\|}=\mathcal{V}_{\perp}$, the excitation energies can also be made same, $\triangle \mathcal{E}_{\|}=\triangle \mathcal{E}_{\perp}$, however the entanglement pressures do always differ. We calculate the entanglement asymmetry, in the same way as (29),

$$
\mathcal{A}_{\text {nonconf }} \equiv \frac{\triangle S_{\perp}-\triangle S_{\|}}{\triangle S_{\perp}+\triangle S_{\|}}=\frac{\beta^{2} \gamma^{2}}{\left(2+\frac{\tilde{\tilde{p}}+3}{\tilde{p}-1} \beta^{2} \gamma^{2}\right)} \leq \frac{\tilde{p}-1}{\tilde{p}+3}
$$


As discussed in the conformal case, the bound gets saturated only in the case of $\mathrm{D} p$ branes having wave like excitations at zero temperature. For this we need to employ the same double limits $\beta \rightarrow 0, \quad z_{0} \rightarrow \infty$, given in (32), on the geometry (41). Thus for nonconformal D-branes with a wave we obtain the asymmetry ratio

$$
\mathcal{A}_{\text {wave }} \equiv \frac{\tilde{p}-1}{\tilde{p}+3}
$$

In conclusion, our results assign maximum entanglement entropy asymmetry to the wave like excitations in a zero temperature CFT. The results can be understood as we now eleborate. The wave like excitations in the CFT at zero temperature generate finite entanglement pressure along the direction of propagation of the wave, while the pressure remains vanishing in all other (transverse) directions. When we switch on finite temperature in the CFT (holographically including black hole in the bulk geometry) some entanglement pressure gets distributed along the transverse directions also. This finite temperature phenomenon reduces the net entanglement entropy asymmetry for the excitations. In the absence of a wave altogether the pressure becomes identical in all directions of the branes and hence entanglement asymmetry would also vanish. Hence the asymmetry in entanglement entropy will necessarily exist if there are uniform wave like excitations or a uniform flow in the CFT. The asymmetry only gets amplified as temperature goes to vanishing values.

\section{Summary}

It has been shown that the entanglement pressure plays a significant role in determining the entanglement entropy for the strip subsytems in the CFT living on the boundary of $A d S_{d+1}$ spacetime. There is an entropy asymmetry along various directions of the CFT if their exists a pressure asymmetry. Besides the entropy asymmetry is directly proportinal to the pressure asymmetry. To quantify this we have determined entanglement asymmetry ratio

$$
\mathcal{A} \equiv \frac{\triangle S_{\perp}-\triangle S_{\|}}{\triangle S_{\perp}+\triangle S_{\|}}=\frac{\beta^{2} \gamma^{2}}{\left(2+\frac{d+3}{d-1} \beta^{2} \gamma^{2}\right)} \leq \frac{d-1}{d+3}
$$

which depends only on the boost parameter $\beta$ and it is bounded. Interestingly the bound is saturated in the large boost limit only (32). Thus a nonzero boost is simply a measure of the entanglement asymmetry. We have discussed a large boost case which is the AdS-wave case. Especially for the AdS waves there exist an optimum entanglement asymmetry

$$
\mathcal{A}_{\text {wave }}=\frac{d-1}{d+3}
$$

which is a universal result at the first order in perturbation analysis. It is independent of

any scale such as energy of wave like excitations $\propto \frac{1}{z_{I}^{d}}$. We expect these results will get corrected by higher orders of perturbation.

In the nonconformal D-branes cases the result gets slightly modified

$$
\mathcal{A}_{\text {nonconf }}=\frac{\beta^{2} \gamma^{2}}{\left(2+\frac{\tilde{p}+3}{\tilde{p}-1} \beta^{2} \gamma^{2}\right)} \leq \frac{\tilde{p}-1}{\tilde{p}+3} \text {. }
$$


The physical relevance of our results is indicated by the fact that the entanglement entropy of subsytems is affected in the presence of boost, or a flow. It is not entirely an unexpected result as the boost indeed represents an asymmetric excitation of the system. It means subsystems along the flow and perpedicular to it get differently entangled as we have determined, $\triangle S_{\perp}>\triangle S_{\|}$. Upto first order this asymmetry is proportional to $\beta^{2}$ (for small velocities). These result however will change at the second order perturbative calculations. Our results however imply more generic situations. Even in the absence of a flow, provided there exists pressure asymmetry in the CFT due to some other reason, the entanglement asymmetry will always arise. The boosted black brane systems are used here only as the known examples to study asymmetric systems. It would be worthwhile to explore other systems like Bianchi models having more generic asymmetry.

Acknowledgments: We are thankful to Arnab Kundu for the discussions.

\section{A The asymptotic expansion for nonconformal black D-branes}

The asymptotic expansion in the Fefferman-Graham coordinates is required to find the energy-momentum tensor of the boundary field theory. The relevant details on holographic renormalization can be found in [15, 16]. Let us define a new holographic coordinate $u$ through

$$
z^{2}=F^{-\frac{4}{\tilde{p}}} u^{2}, \quad F=1+\frac{u^{\tilde{p}}}{u_{0}^{\tilde{p}}}, \quad u_{0}^{\tilde{p}} \equiv 4 z_{0}^{\tilde{p}}
$$

In these $u$ coordinates an expansion of (41) in the neighborhood of UV boundary $(u=0)$ becomes

$$
\begin{aligned}
& d s^{2} \simeq g_{\text {eff }}\left[\frac { 1 } { u ^ { 2 } } \left[\left(-1+4\left(\frac{\tilde{p}-1}{\tilde{p}}+\beta^{2} \gamma^{2}\right) \frac{u^{\tilde{p}}}{u_{0}^{\tilde{p}}}+\cdots\right) d t^{2}+\left(1+4\left(\frac{1}{\tilde{p}}+\beta^{2} \gamma^{2}\right) \frac{u^{\tilde{p}}}{u_{0}^{\tilde{p}}}+\cdots\right) d y^{2}\right.\right. \\
&\left.\left.-\frac{8 \beta \gamma^{2} u^{4}}{u_{0}^{\tilde{p}}} d t d y+\left(1+\frac{4}{\tilde{p}} \frac{u^{\tilde{p}}}{u_{0}^{\tilde{p}}}+\cdots\right)\left(d x_{2}^{2}+\cdots+d x_{p}^{2}\right)\right]+\frac{4}{(5-p)^{2}} \frac{d u^{2}}{u^{2}}+d \Omega_{8-p}^{2}\right] \\
& \equiv g_{\text {eff }}\left[\frac{1}{u^{2}}\left(\eta_{\alpha \beta}+t_{\alpha \beta} u^{\tilde{p}}+\cdots\right) d x^{\alpha} d x^{\beta}+\frac{4}{(5-p)^{2}} \frac{d u^{2}}{u^{2}}+d \Omega_{8-p}^{2}\right]
\end{aligned}
$$

The last line in the above equation indicates that the spacetime geometry is expanded in asymptotic neighborhood of conformally $A d S_{p+2} \times S^{8-p}$ spacetime. Besides in these coordinates, $u$ coincides with the energy scale of the $A d S_{p+2}$ geometry. The $\eta_{\alpha \beta}$ is flat Minkowski metric with index $\alpha=0,1,2, \cdots, p$. The effective coupling has the FG expansion (RG flow) given by

$$
g_{e f f}=\frac{\left(l_{p} u^{3-p}\right)^{\frac{1}{5-p}}}{F^{\frac{3-p}{7-p}}} \simeq\left(l_{p} u^{3-p}\right)^{\frac{1}{5-p}}\left(1-\frac{3-p}{7-p} \frac{u^{\tilde{p}}}{u_{0}^{\tilde{p}}}+\cdots\right)
$$


In $p=3$ (conformal) case the $g_{\text {eff }}$ however remains fixed. The important point to notice from the FG expansion is that the overall conformal factor of the string metric (57) and the string coupling $e^{\phi}$ (given in (41)) are both governed by the fluctuations of the single quantity $g_{\text {eff }}$. The fluctuations of the dilaton field, $\delta \phi$, can also be obtained from the expression

$$
\begin{aligned}
e^{\phi} & =\frac{(2 \pi)^{2-p}}{d_{p} N}\left(l_{p} u^{3-p}\right)^{\frac{\tilde{p}}{4}}\left(1-\frac{3-p}{2} \frac{u^{\tilde{p}}}{u_{0}^{\tilde{p}}}+\mathcal{O}\left(u^{2 \tilde{p}}\right)\right) \\
& \equiv e^{\phi_{0}}\left(1+\delta \phi_{(\tilde{p})} u^{\tilde{p}}+\cdots\right)
\end{aligned}
$$

where $\phi_{0}$ represents the dilaton field in the absence of the excitations. The first order fluctuation of dilaton are thus $\delta \phi_{(\tilde{p})}=-\frac{3-p}{2} \frac{1}{u_{0}^{\tilde{p}}}$. Obviously $\delta \phi_{(\tilde{p})}$ has opposite signs for $p>3$ and $p<3$ branes. (For D3 brane $\delta \phi_{(\tilde{p})}$ vanishes as it should be for $4 D$ conformal field theory.) The nonvanishing components of stress-energy tensor of the boundary theory can now be obtained from the expression within the angular brackets in asymptotic expansion (57)

$$
\begin{aligned}
& t_{00}=\left(\frac{\tilde{p}-1}{\tilde{p}}+\beta^{2} \gamma^{2}\right) \frac{4}{u_{0}^{\tilde{p}}}, \quad t_{y y}=\left(\frac{1}{\tilde{p}}+\beta^{2} \gamma^{2}\right) \frac{4}{u_{0}^{\tilde{p}}} \\
& t_{0 y}=\beta \gamma^{2} \frac{4}{u_{0}^{\tilde{p}}}, \quad t_{i i}=\frac{1}{\tilde{p}} \frac{4}{u_{0}^{\tilde{p}}}, \quad(i=2,3, \ldots, p)
\end{aligned}
$$

The tensor $t_{\alpha \beta}$ has a nonvanishing trace. It is worthwhile to observe that the trace, $t_{\alpha}^{\alpha}$, and $\delta \phi$ have a relationship

$$
\frac{1}{4} t_{\alpha}^{\alpha}-\frac{3-p}{7-p} \delta \phi_{(\tilde{p})}=0
$$

as they both depend on single deformation parameter $u_{0}$. Actually this relation follows from Ward identities in holographic renormalization of the boundary theory [19]. Also $\nabla_{\alpha} t^{\alpha \beta}=0$ trivially. We should not be checking them over here as these are automatic in the FG expansion (57) of nonextremal geometry. The energy of the excitations above the extremality for the boosted solutions is then given by

$$
\triangle \mathcal{E}=\frac{V_{p} \Theta_{8-p} Q_{p}}{16 \pi G_{N}}\left(\frac{\tilde{p}-1}{\tilde{p}}+\beta^{2} \gamma^{2}\right) \frac{7-p}{z_{0}^{\tilde{p}}}
$$

where $V_{p}$ is the $p$-dimensional spatial volume of all $x_{i}$ 's and $Q_{p}$ is a combinatoric factor defined earlier. $\Theta_{8-p}$ is unit volume of the $S^{8-p}$, and $G_{N}$ is the Newton's constant in ten dimensions. Similarly pressure components along the boost and in perpedicular directions are

$$
\begin{aligned}
& \triangle \mathcal{P}_{\|}=\triangle \mathcal{P}_{y}=\frac{\Theta_{8-p} Q_{p}}{16 \pi G_{N}}\left(\frac{1}{\tilde{p}}+\beta^{2} \gamma^{2}\right) \frac{7-p}{z_{0}^{\tilde{p}}} \\
& \triangle \mathcal{P}_{\perp}=\triangle \mathcal{P}_{x_{2}}=\frac{\Theta_{8-p} Q_{p}}{16 \pi G_{N}} \frac{7-p}{\tilde{p} z_{0}^{\tilde{p}}}=\triangle \mathcal{P}_{x_{3}}=\cdots
\end{aligned}
$$




\section{References}

[1] J. Maldacena, Adv. Theor. Math. Phys. 2, 231 (1998) arXiv:9711200 [hep-th]; S. Gubser, I Klebanov and A.M. Polyakov, Phys. lett. B 428, 105 (1998) arXiv:9802109[hep-th]; E. Witten, Adv. Theor. Math. Phys. 2, 253 (1998) arXiv: 9802150 [hep-th].

[2] S. Ryu and T. Takayanagi, Phys. Rev. Lett. 86 (2006) 181602, arXiv:0603001[hep-th]; S. Ryu and T. Takayanagi, JHEP 0608 (2006) 045, arXiv:0605073[hep-th].

[3] N. Ogawa, T. Takayanagi, and T. Ugajin, JHEP 1201 (2012) 125, arXiv: 1111.1023 [hep-th].

[4] J. Bhattacharya, M. Nozaki, T. Takayanagi and T. Ugajin, "Thermodynamical Property of Entanglement Entropy for Excited States," Phys. Rev. Lett. 110, no. 9, 091602 (2013) [arXiv:1212.1164.

[5] D. Allahbakhshi, M. Alishahiha and A. Naseh, "Entanglement Thermodynamics," JHEP 1308, 102 (2013) [arXiv:1305.2728 [hep-th]].

[6] D. W. Pang, "Entanglement thermodynamics for nonconformal D-branes," Phys. Rev. D 88 (2013) 12, 126001 [arXiv:1310.3676 [hep-th]].

[7] C. Park, "Holographic entanglement entropy in the nonconformal medium," Phys. Rev. D 91, no. 12, 126003 (2015) [arXiv:1501.02908 [hep-th]].

[8] G. Wong, I. Klich, L. A. Pando Zayas and D. Vaman, JHEP 1312, 020 (2013) arXiv:1305.3291 [hep-th]]; S. He, J. R. Sun and H. Q. Zhang, arXiv:1411.6213 [hep-th]; S. Chakraborty, P. Dey, S. Karar and S. Roy, JHEP 1504, 133 (2015) arXiv:1412.1276 [hep-th]]; N. Kim and J. H. Lee, "Time-evolution of holographic entanglement entropy and metric perturbations," arXiv:1512.02816 [hep-th].

[9] R. Mishra and H. Singh, "Perturbative entanglement thermodynamics for AdS spacetime: Renormalization," JHEP 1510 (2015) 129 [arXiv:1507.03836 [hep-th]].

[10] M. Nozaki, T. Numasawa, A. Prudenziati and T. Takayanagi, "Dynamics of Entanglement Entropy from Einstein Equation," Phys. Rev. D 88, no. 2, 026012 (2013) arXiv:1304.7100 [hep-th]].

[11] D. D. Blanco, H. Casini, L. Y. Hung and R. C. Myers, "Relative Entropy and Holography," JHEP 1308, 060 (2013) arXiv:1305.3182 [hep-th]].

[12] V. E. Hubeny, M. Rangamani and T. Takayanagi, "A Covariant holographic entanglement entropy proposal," JHEP 0707, 062 (2007) [arXiv:0705.0016 [hep-th]]. 
[13] H. Singh, "Special limits and non-relativistic solutions," JHEP 1012, 061 (2010) arXiv:1009.0651[hep-th]]; H. Singh, "Lifshitz/Schródinger Dp-branes and dynamical exponents," JHEP 1207, 082 (2012) [arXiv:1202.6533 [hep-th]].

[14] K. Narayan, T. Takayanagi and S. Trivedi, JHEP 04 (2013) 051, arXiv: 1212.4328 [hep-th].

[15] V. Balasubramanian and P. Kraus, "A Stress tensor for Anti-de Sitter gravity," Commun. Math. Phys. 208, 413 (1999) [hep-th/9902121]; P. Kraus, F. Larsen and R. Siebelink, "The gravitational action in asymptotically AdS and flat space-times," Nucl. Phys. B 563, 259 (1999) (hep-th/9906127); M. Bianchi, D. Z. Freedman and K. Skenderis, "Holographic renormalization," Nucl. Phys. B 631, 159 (2002) [hep-th/0112119].

[16] I. Kanitscheider, K. Skenderis, M. Taylor, "Precision holography for non-conformal branes," arXiv: 0807.3324[hep-th].

[17] R. Mishra and H. Singh, to appear.

[18] N. Itzhaki, J.M. Maldacena, J. Sonnenschein and S. Yankielowicz, Phys. Rev. D58 046004 (1998), arXiv: 9802042 [hep-th].

[19] H.J. Boonstra, K. Skenderis and P.K. Townsend, "The domain-wall/QFT correspondence", JHEP 9901, 003 (1999), arXiv: 9807137 [hep-th]. 